University of Wollongong

Research Online

Faculty of Engineering and Information

Faculty of Engineering and Information

Sciences - Papers: Part A

Sciences

$1-1-2014$

Degradation trend estimation and prognosis of large low speed slewing bearing lifetime

Prabuono Buyung Kosasih

University of Wollongong, buyung@uow.edu.au

Wahyu Caesarendra

University of Wollongong,wc026@uowmail.edu.au

Kiet Tieu

University of Wollongong, ktieu@uow.edu.au

Achmad Widodo

Diponegoro University

Craig A. S Moodie

University of Wollongong, cam920@uow.edu.au

Follow this and additional works at: https://ro.uow.edu.au/eispapers

Part of the Engineering Commons, and the Science and Technology Studies Commons

Research Online is the open access institutional repository for the University of Wollongong. For further information contact the UOW Library: research-pubs@uow.edu.au 


\title{
Degradation trend estimation and prognosis of large low speed slewing bearing lifetime
}

\author{
Abstract \\ In many applications, degradation of bearing conditions is usually monitored by changes in time-domain \\ features. However, in low speed $(<10 \mathrm{rpm}$ ) slewing bearing, these changes are not easily detected \\ because of the low energy and low frequency of the vibration. To overcome this problem, a combined low \\ pass filter (LPF) and adaptive line enhancer (ALE) signal preconditioning method is used. Time-domain \\ features such as root mean square (RMS), skewness and kurtosis are extracted from the output signal of \\ the combined LPF and ALE method. The extracted features show accurate information about the incipient \\ of fault as compared to extracted features from the original vibration signal. This information then \\ triggers the prognostic algorithm to predict the remaining lifetime of the bearing. The algorithm used to \\ determine the trend of the nonstationary data is auto-regressive integrated moving average (ARIMA).

\section{Keywords} \\ speed, low, large, prognosis, estimation, trend, degradation, lifetime, bearing, slewing \\ Disciplines \\ Engineering | Science and Technology Studies

\section{Publication Details} \\ Kosasih, P., Caesarendra, W., Tieu, A. K., Widodo, A. \& Moodie, C. A. S. (2014). Degradation Trend \\ Estimation and Prognosis of Large Low Speed Slewing Bearing Lifetime. Applied Mechanics and \\ Materials, 493 343-348.
}




\title{
Degradation Trend Estimation and Prognosis of Large Low Speed Slewing Bearing Lifetime
}

\author{
Wahyu Caesarendra ${ }^{1, a}$,Buyung Kosasih ${ }^{1, b}$,Kiet Tieu ${ }^{1, \mathrm{c}},{ }^{\text {,Achmad Widodo }}{ }^{2, \mathrm{~d}}$ and \\ Craig A.S. Moodie ${ }^{1, \mathrm{e}}$ \\ ${ }^{1}$ School of Mechanical, Materials and Mechatronic Engineering, University of Wollongong, \\ Wollongong, NSW 2522, Australia \\ ${ }^{2}$ Mechanical Engineering Department, Diponegoro University, Semarang 50275, Indonesia \\ awc026@uowmail.edu.au, bbuyung@uow.edu.au, ${ }^{\mathrm{c} k t i e u @ u o w . e d u . a u, ~}{ }^{\mathrm{d}}$ awid@undip.ac.id, \\ ecasmoodie@bigpond.com
}

Keywords: Adaptive line enhancer, Autoregressive integrated moving average, Degradation trend, Prognosis, Slewing bearing

\begin{abstract}
In many applications, degradation of bearing conditions is usually monitored by changes in time-domain features. However, in low speed $(<10 \mathrm{rpm})$ slewing bearing, these changes are not easily detected because of the low energy and low frequency of the vibration. To overcome this problem, a combined low pass filter (LPF) and adaptive line enhancer (ALE) signal pre-conditioning method is used. Time-domain features such as root mean square (RMS), skewness and kurtosis are extracted from the output signal of the combined LPF and ALE method. The extracted features show accurate information about the incipient of fault as compared to extracted features from the original vibration signal. This information then triggers the prognostic algorithm to predict the remaining lifetime of the bearing. The algorithm used to determine the trend of the non-stationary data is auto-regressive integrated moving average (ARIMA).
\end{abstract}

\section{Introduction}

Slewing bearingsare typically used in heavy machinery to support high axial and radial loads. In steel mills, slewing bearings are often critical production items. An unplanned downtime when a bearing breaks down can be very expensive due to the loss of production. As a replacement large slewing bearing can take a few months to arrive due to the long lead time in manufacturing and delivery, steel mills often carry spare bearings to guard against these unforeseen circumstances adding an extra cost. In order to prevent unplanned downtime from occurring, a prognostic method for predicting the degradation of slewing bearings is needed. However, to date only limited research has been reported on the condition monitoring and prediction of the slewing bearing lifetime [1-3]. While there are three types of methods for the prognosis of the slewing bearing - oil-based [2], numerical [3] and vibration-based - this paper is concerned only with vibration.

Although vibration prognostic analysis has been developed and applied widely in medical science fields, it still presents a major challenge in mechanical engineering and the area of maintenance. In this field, vibration prognostic analysis has been mainly applied to rotating element bearing under high and medium rotating speed, and while condition monitoring and fault diagnosis of low speed rolling element bearing have been reported [4], the methods have seldom been applied to low speed slewing bearings. This is because slewing bearings operate at slow speeds (0.5 to $10 \mathrm{rpm})$, in continuous or intermittent modes, in a partially reversible direction of rotation under high mechanical load, which makes it difficult to analyze the vibration signal.

In practice, the degradation of rotating machinery is often monitored via time-domain features, such as root mean square (RMS), kurtosis, skewness, and entropy [5]. Unfortunately for low speed slewing bearing, the changes in these features cannot be identified due to the low energy and low frequency of the vibration.

This paper proposes a prognostic technique for low speed slewing bearing based on combined low pass filter (LPF) and adaptive line enhancer (ALE)as a pre-conditioning method, and auto-regressive 
integrated moving average (ARIMA) as a prediction method. The ARIMA model with selected lags and adaptively modified coefficients for time-series forecasting of the slewing bearing lifetime is developed.The proposed technique provides an accurate degradation trend prediction of the bearing life for a slewing bearing case.

\section{Signal Pre-conditioning}

The vibration signal characteristic of a low speed slewing bearing is different to the signal of a high speed rotationalroller bearing. For a slewing bearing, the wear on the contacting part between roller and raceway occurs slowly due to the long period of high load condition. However, if there is contamination ingress then the wear process is accelerated and deterioration of the bearing occurs more rapidly. When the incipient wear of single defect occur, for instance on a roller element, the contact between the defect spot and raceway will induce impact energy. This impact energy will excite the resonance frequency of the bearing or machine and generate an impulse signal. This impulse signal will repeat periodically each time the defect spot rolls over the raceway. Due to the very low rotational speed of approximately $1 \mathrm{rpm}$ the generated energy is very low and deeply masked by the background noise.

In practice, a single defect condition rarely occurs. In reality, as dust contamination spreads inside the bearing, rollers and raceways can experience simultaneous incipient wear or defect. Accumulation of multiple worn spots/defects will generate a high frequency signal and bury the low frequency-low energy signal. Therefore, the challenge in monitoring the condition of the slewing bearing in relation to the vibration signal is how to separate the low frequency-low energy signal from the high frequency signal. This is difficult to achieve using frequency domain analysis such as FFT. To overcome this problem, a pre-conditioning step to extract the information about the progression of the deterioration level from the vibration signal is required.In this paper, a combined LPF and ALE is used for the pre-conditioning stage prior to the features calculation stage.

\section{Prognostic Analysis}

Degradation Trend Estimation.In this paper, the degradation trend is simply calculated using a cumulative technique,

$$
Z_{t+1}=\sum_{i=1}^{t} r_{t}
$$

where $r$ is individual feature, $Z$ represents the degradation trend and $t$ is the number of day. It should be noted that individual feature, $r$ is the extracted features after the pre-conditioned signal stage.

Auto-regressive Integrated Moving Average (ARIMA).The auto-regressive integrated moving average (ARIMA)is a forecasting method for non-stationary data with trend behavior [6]. The first step in ARIMA is transforming non-stationary data into linear form by using differencing technique. After the differencing process, the result becomes a stationary time series. Therefore, in ARIMA forecasting the prediction or future value is supposed to be a linear combination of past values and past errors, where the auto-regressive (AR) variable is a past value, and moving average (MA) variable is a past error from historical data. According to [7], the general non-stationary ARIMA model which number of difference, $d=1$ is expressed as

$$
\begin{aligned}
& \text { Autoregressive factor: } \phi_{1-p}(B)=1-\phi_{1} B(1)-\phi_{2} B(2)-\ldots-\phi_{p} B(p) \\
& \text { Moving average factor: } \theta_{1-q}(B)=1-\theta_{1} B(1)-\theta_{2} B(2)-\ldots-\theta_{q} B(q)
\end{aligned}
$$

where, $p$ is the number of auto-regressive termsand $q$ is the number of lagged forecast errors in the prediction equation. 
Prognosis Algorithm.The prognostic method is depicted in Fig. 1. The next stage after the pre-conditioning stage, features extraction and degradation estimation is the prediction of the final failure. Threetime-domain features are extracted after LPF+ALE pre-conditioning to reveal the information of the bearing condition. Cumulative technique of each feature is estimated to show the degradation trend and incipient failure information. Using the incipient failure information, ARIMA is used to forecast the final failure.

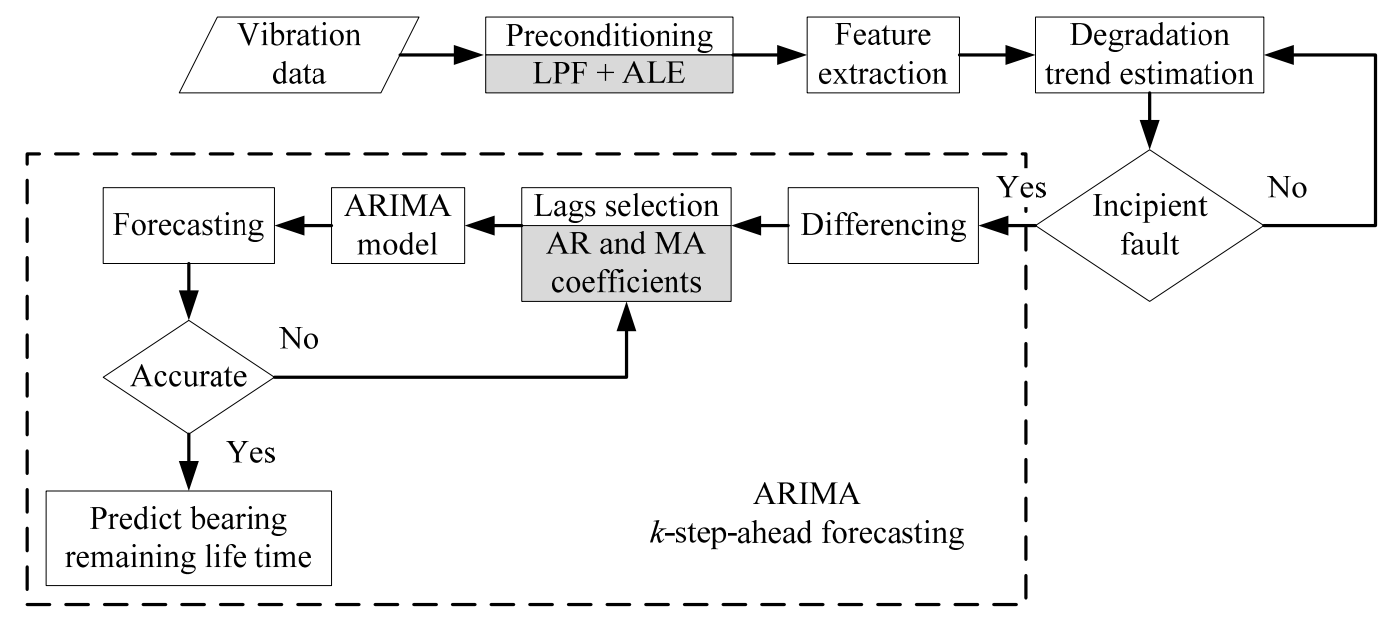

Fig. 1 Prognosis based ARIMA $k$-step-ahead forecasting

The ARIMA forecasting method shown in Fig. 1 follows several steps:

Step 1 (Check stationary): The degradation trend data will be made stationary before being fed into the prognostic stage. If the data is not stationary, it needs to be transformed into stationary data using the differencing technique.

Step 2 (Initial structures selection): Select the auto-regression (AR) and moving average (MA) structures using partial autocorrelation function (PACF) and autocorrelation function (ACF) as the initial forecasting model.

Step 3 (Coefficients estimation): Estimate the AR and MA coefficients based on the selected structures from step 2 using least-square regression.

Step 4 (Forecasting): Based on the forecasting model (with the selected structures and estimated coefficients), multi-step-ahead prediction is then conducted to forecast the final failure time.

Step 5 (Refine the model): If the predictions result in an unexpected trend, repeat step 2 with trial structures and continue to step 3. In step 4 the multi-step-ahead forecasting applies to the adaptively modified AR and MA coefficients at every one-step-ahead forecasting.

\section{Experimental Setup}

The basic requirement of a slewing bearing test-rig is to run a new bearing to the stage when the bearing needs to be replaced under controlled continuous running conditions. Fig. 2 shows the schematic of slewing bearing test-rig showing how the bearing is attached and sensors attachment. The test-rig can be operated at 1 to $12 \mathrm{rpm}$. The test-rig was designed to meet the real conditions in a steel making company. In this project, the test-rig was operated in one direction of rotation at $1 \mathrm{rpm}$. The slewing bearing used was an axial/radial bearing supplied by Schaeffler (INA YRT260).

Four Accelerometers were placed on the inner radial surface at 90 deg to each other.The type of accelerometer used is IMI608 A11 ICP type sensor. The accelerometers were connected to high speed DAQ (PS3424) from Picoscope. This DAQ device was used with PICO software for real time data viewing/analysis, including saving data to the *.txt file.Each day two vibration test samples were gathered. The slewing bearing test-rig was operated in a noisy environment; therefore, the acquisition 
was conducted at two different times: at mid-day (with $600 \mathrm{~Hz}$ ) sampling rates and at mid-night (with $4880 \mathrm{~Hz}$ ) sampling rates. The vibration data was acquired from February to August 2007 (138 days). In order to accelerate the bearing defect, dust contamination was injected into the bearing in the middle of April 2007. The constant load of 30tonne was applied via hydraulic pressure as shown in Fig. 2.

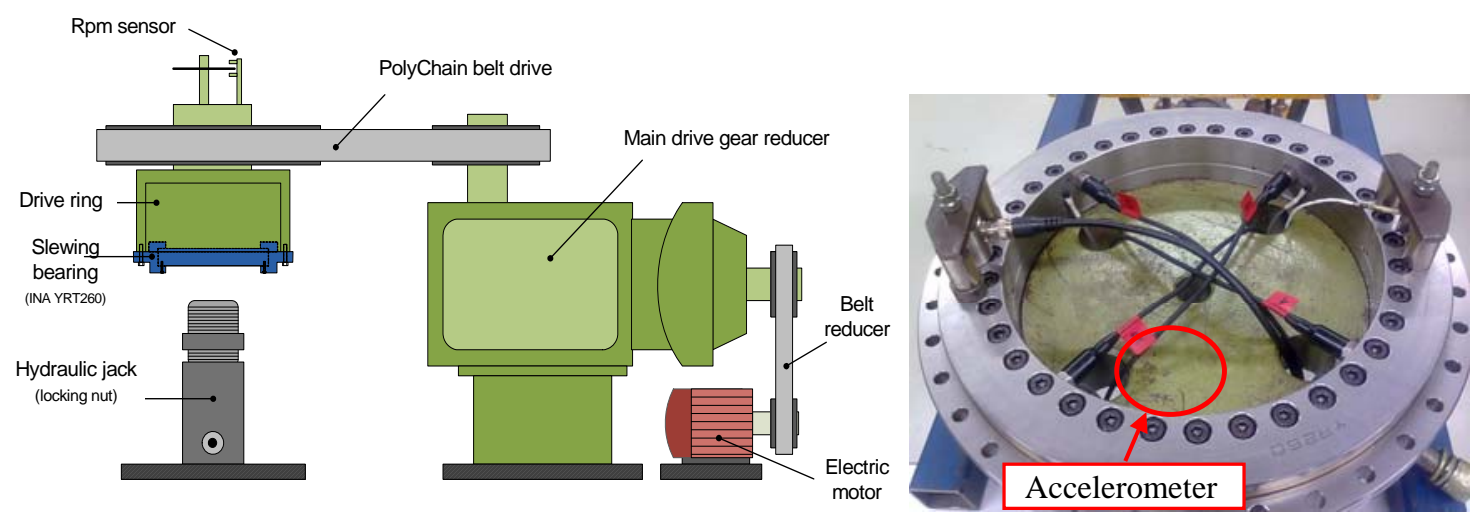

Fig. 2(a) Schematic of slewing bearing test-rig; (b) image of sensors attachment

\section{Result and Discussions}

Filtering process and degradation trend estimation.In this study RMS, skewness and kurtosis are utilized. These features had shown an accurate result in distinguishing several conditions of induction motor including normal and different fault types [8].

The three time-domain features mentioned above were initially extracted from raw vibration data. These features represent the degradation condition of bearing from health condition (in February) to bearing collapse (in August), however the incipient fault is shown more clearly using extracted features of LPF+ALE. This is because features are extracted from high frequency accumulated signals instead of a bearing fault signal. Therefore LPF is used as the first filtering step. The LPF used the eight orders Butterworth with a cut-off frequency of $40 \mathrm{~Hz}$. Further, ALE based on the concept of linear prediction can be used to separate the non-periodic and the periodic signal. In this case, the bearing defect signal is assumed as nearly periodic. And because any signal except the defect signal is non-periodic, it can be expected from ALE that the true fault signal can be separated from the noise. By calculating the features of this signal the appropriate information about the bearing condition can be extracted. A built-in MATLAB function of the block LMS FIR adaptive filter algorithm is used with the signal delay, $\Delta=100$, filter length, $L=32$ and number of blocks, $N=32$.

Figs. 4(a) to (c) show the comparison between extracted features from raw vibration data and that from LPF+ALE result. It can be seen that the features of LPF+ALE results show a consistency in representing the bearing condition.

ARIMA forecasting. According to the extracted features, the degradation trend of kurtosis is selected as the prediction object and normalized from 0 (represents good condition) to 1 (represents final failure). The total days from 21 February 2007 to 31 August 2007 are 138 days. This paper demonstrates an ARIMA forecasting algorithm based on the econometric toolbox of MATLAB with selected lags and adaptively modified AR and MA coefficients. According to Figs. 4(a) to (c) the incipient failure is 90; however data up to day 120 was used as a training data to estimates the forecasting model. The ARIMA model based on the training data is obtained as

Autoregressive factor: $\phi_{1,5,7}(B)=1-\phi_{1} B^{1}+\phi_{5} B^{5}+\phi_{7} B^{7}$

Moving average factor: $\theta_{1}(B)=1-\theta_{1} B^{1}$ 
Two expressions above can be written as ARIMA $(7,1,2)$ model. The posterior value from day 121 to 138 is predicted using multi-step-ahead forecasting method by adaptively modifying the AR and MA coefficients every one-step-ahead.The illustration of adaptive ARIMA forecasting is shown in Fig. 3. The ARIMA prediction result is shown in Fig. 4(d). The accuracy of final failure prediction for ARIMA is evaluated using the following expression

$$
\text { Accuracy }=\left(1-\frac{\left|t_{a}-t_{p}\right|}{t_{a}}\right) \times 100 \%=97.83 \%
$$

where $t_{a}$ is actual final failure (138 days) and $t_{p}$ denotes the prediction of final failure (135 days).

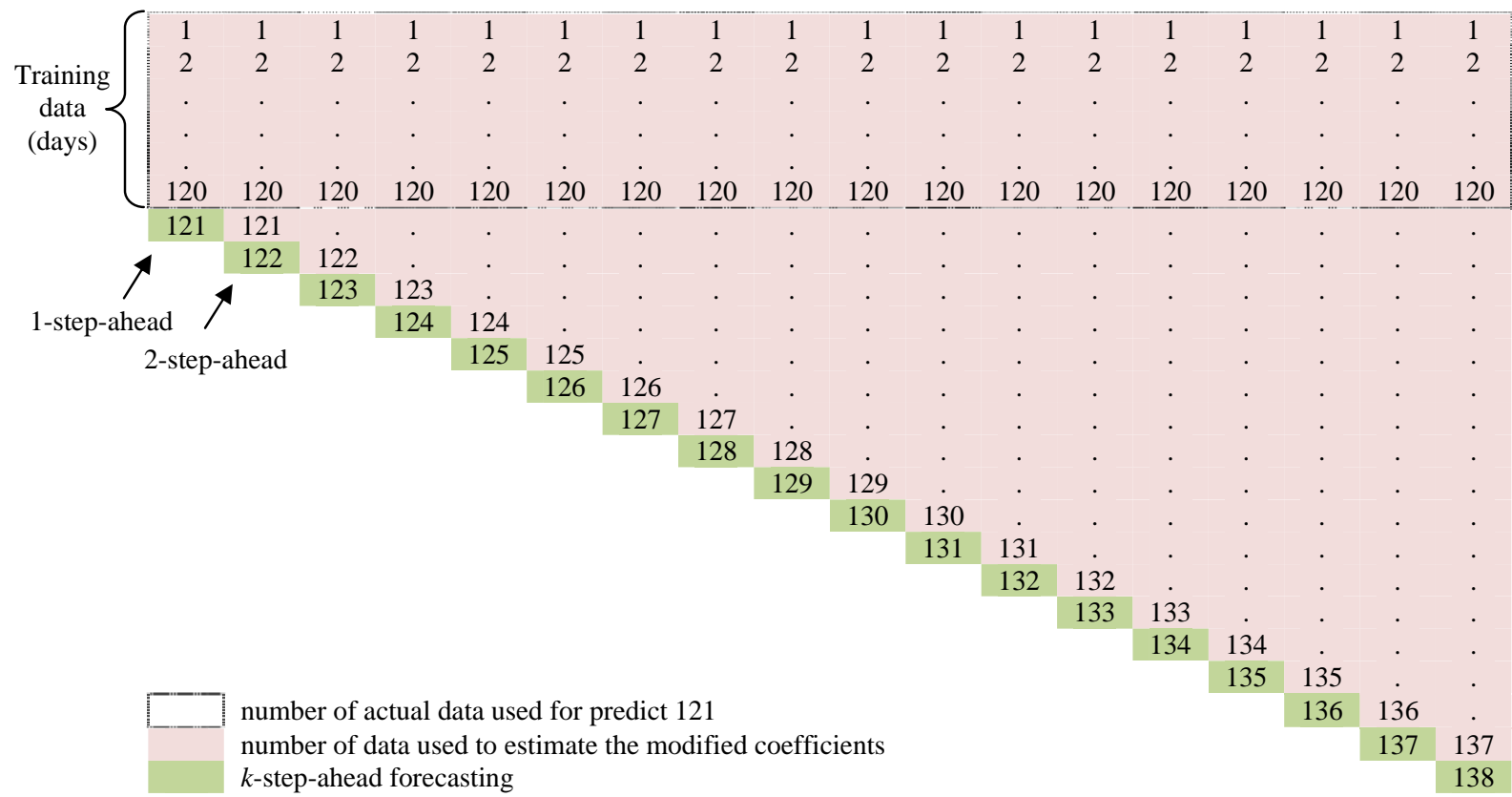

Fig. 3 Illustration of ARIMA $k$-step-ahead forecasting based on adaptively modified coefficients

\section{Summary}

The non-stationary time series prognostic technique for prediction of bearing life time based multi-step-ahead forecasting has been presented. The components of the prognostic technique can be described as: preconditioning method, time-domain features extraction, degradation trend estimation and ARIMA forecasting. The pre-conditioning method has to be used to remove the high frequencies signal and the non-periodic signal. The time-series features extraction and the degradation trend estimation are used to reveal the information about the deterioration of the slewing bearing. The final failure prediction based ARIMA was developed. It was shown that this technique can predict the deterioration trend from incipient to final failure. The selected lags and adaptively AR and MA coefficients show an accurate prediction result.

\section{Acknowledgment}

The first author gratefully acknowledges the financial support provided by the University of Wollongong through the University Postgraduate Award (UPA) and International Postgraduate Tuition Award (IPTA). 

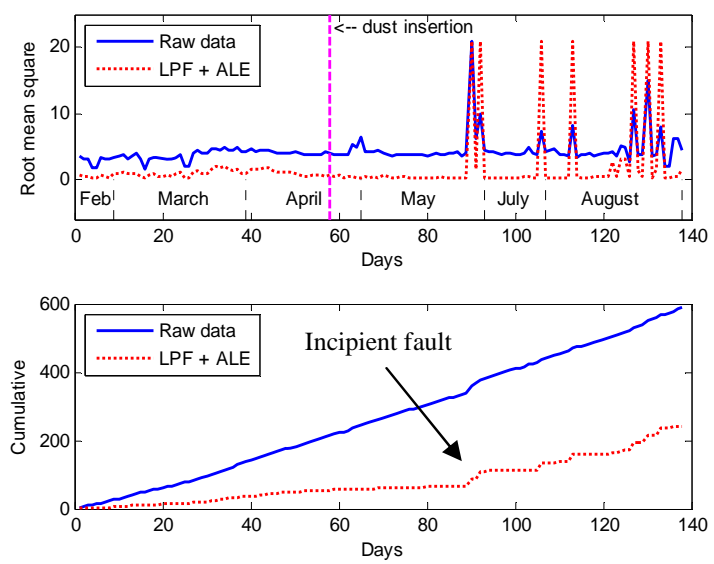

(a)
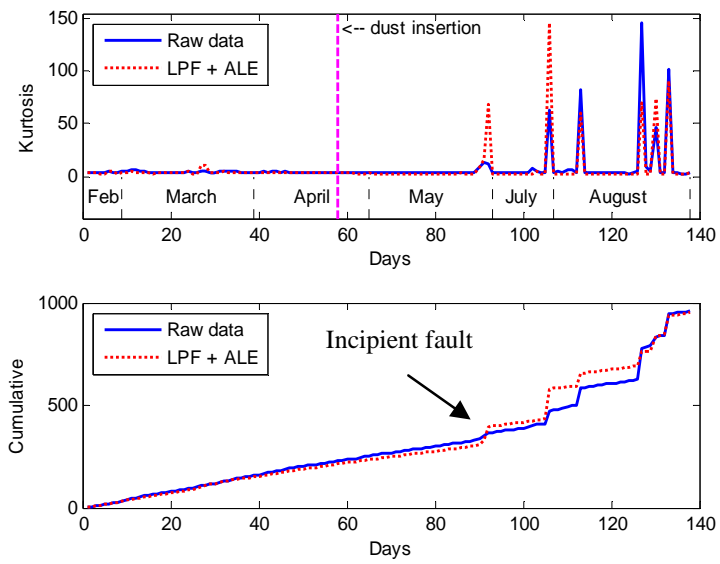

(c)
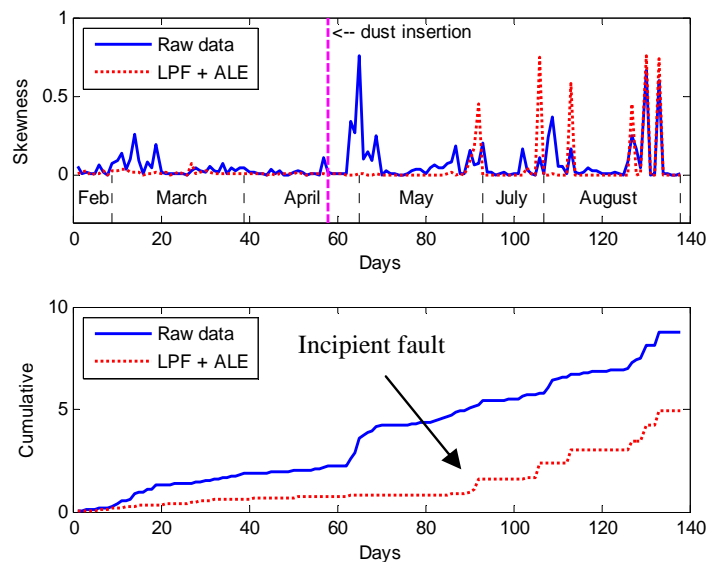

(b)

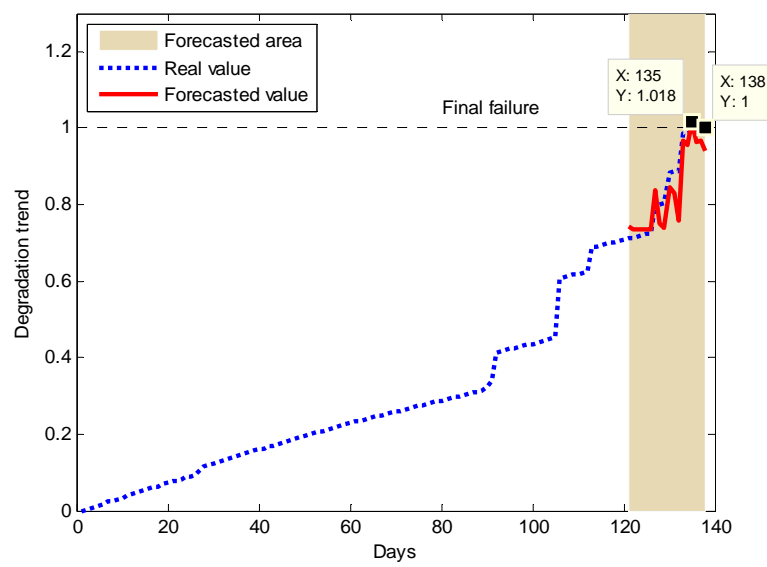

(d)

Fig. 4 (a) RMS feature and degradation trend of RMS feature, (b) Skewness feature and degradation trend of skewness feature, (c) Kurtosis featureand degradation trend of kurtosis feature, (d) ARIMA $k$-step-ahead forecasting result.

\section{References}

[1] C.A.S. Moodie,An investigation into the condition monitoring of large slow speed slew bearings, PhD Thesis in Faculty of Engineering. 2009, University of Wollongong: Wollongong.

[2] X. Bai, H. Xiao, L. Zhang, The condition monitoring of large slewing bearing based on oil analysis method, Key Eng. Mater. 474-476 (2011) 716-719.

[3] S. Glödez, R. Potocnik, J. Flasker,Computational model for calculation of static capacity and lifetime of large slewing bearing's raceway, Mech. Mach. Theory 47 (2012) 16-30.

[4] C.K. Mechefske, J. Mathew, Fault detection and diagnosis in low speed rolling element bearings Part I: The use of parametric spectra, Mech. Syst. Signal Process. 6 (1992) 297-307.

[5] B.S Yang, A. Widodo, Introduction of Intelligent Machine Fault Diagnosis and Prognosis, Nova Science Publishers, Inc., USA, 2009.

[6] G.B.P. Box, G.M. Jenkins, G.C. Reinsel, Time Series Analysis, Forecasting and Control, $3^{\text {rd }}$ ed., Prentice Hall, Englewood Cliffs, NJ, 1994.

[7] W.W.S. Wei, Time Series Analysis: Univariate and Multivariate Methods, $2^{\text {nd }}$ ed., Pearson Education, USA, 2006.

[8] A. Widodo, B.S. Yang, Support vector machine in machine condition monitoring and fault diagnosis, Mech. Syst. Signal Process. 21 (2007) 2560-2574. 